\title{
Long non-coding RNA AFAP1-antisense RNA 1 promotes the proliferation, migration and invasion of gastric cancer cells and is associated with poor patient survival
}

\author{
HUAZHOU ZHAO ${ }^{1,2^{*}}$, KECHENG ZHANG $^{1 *}$, TING WANG $^{3 *}$, JIANXIN CUI $^{1}$, HONGQING XI $^{1}$, \\ YI WANG $^{1}$, YANJING SONG ${ }^{1}$, XUDONG ZHAO $^{1}$, BO WEI $^{1}$ and LIN CHEN $^{1}$ \\ ${ }^{1}$ Department of General Surgery, General Hospital of Chinese People's Liberation Army, Beijing 100853; \\ ${ }^{2}$ Department of General Surgery, 309th Hospital of Chinese People's Liberation Army, Beijing 100091; \\ ${ }^{3}$ Medical Administration Division of Beijing Chaoyang Hospital, Beijing 100020, P.R. China
}

Received October 11, 2016; Accepted November 21, 2017

DOI: $10.3892 / \mathrm{ol} .2018 .8389$

\begin{abstract}
Gastric cancer (GC) is the second-leading cause of cancer-associated mortality worldwide. AFAP1-antisense RNA 1 (AFAP1-AS1), a long non-coding RNA (lncRNA), is believed to promote the aggressive progression of cancer; however, its role in GC remains largely unknown. In the present study, the expression of AFAP1-AS1 in GC tissues and cell lines was measured using reverse transcription-quantitative polymerase chain reaction (RT-qPCR). Knockdown of AFAP1-AS1 was performed using a lentiviral vector containing a short hairpin RNA. The proliferation of GC cells was measured using Cell Counting kit-8. The migration and invasion of GC cells were analyzed using a QCM Laminin Migration Assay kit and a Cell Invasion Assay kit. The levels of epithelial-mesenchymal transition (EMT)-associated proteins were detected by western blot analysis. The cut-off value of the expression of AFAP1-AS1 was evaluated using receiver operating characteristic (ROC) curves and patient survival rate was analyzed using Kaplan-Meier. The expression of AFAP1-AS1 was significantly increased in the primary tumor tissues of GC patients with lymph node metastasis or tumor node metastasis stage (stage III or IV; P<0.01). ROC curve analysis revealed that the expression of AFAP-AS1, at a cut-off value of 0.5040 , could distinguish GC tissues from the matched normal tissues, with an AUC of 0.8802 , sensitivity of $81.25 \%$ and specificity of $83.75 \%$. The overexpression of
\end{abstract}

Correspondence to: Dr Bo Wei or Dr Lin Chen, Department of General Surgery, General Hospital of Chinese People's Liberation Army, 28 Fuxing Road, Beijing 100853, P.R. China

E-mail: weibobj301@sina.com

E-mail: chenlinbj@sina.com

*Contributed equally

Key words: AFAP1-antisense RNA 1, gastric cancer, epithelialmesenchymal transition, proliferation, metastasis
AFAP1-AS1 was positively associated with the poor survival rates of GC patients. Furthermore, the downregulation of AFAP1-AS1 significantly inhibited the proliferation, migration and invasion of GC cells in vitro $(\mathrm{P}<0.01)$. The decrease in AFAP1-AS1 expression significantly suppressed the expression level of $\mathrm{N}$-cadherin protein in GC cells and increased that of E-cadherin. The present study demonstrated that the expression signature of AFAP1-AS1 may serve as a biomarker for the diagnosis and prognosis of GC, and its downregulation may repress the aggressive progression of GC, partially through inhibiting the EMT progress.

\section{Introduction}

Gastric cancer (GC) is the second most commonly diagnosed cancer in men and fourth most commonly diagnosed cancer in women, and is the second-leading cause of cancer-associated mortality in China (1). Although the incidence and mortality rates of GC have significantly decreased, a large number of newly diagnosed cases have occurred as a result of population growth and the ageing population (1). Tumor cell metastasis is a major cause of GC-associated mortality and markedly decreases the efficacy of treatment (2). An increasing number of oncogenes and tumor suppressors were observed to be involved in the progression of GC (2); however, the mechanism underlying GC metastasis remains largely unclear.

Long non-coding RNAs (lncRNAs), a class of non-coding RNAs that do not code for proteins, are $>200$ nucleotides in length (3). The aberrant expression of lncRNAs is believed to be associated with the aggressive development of cancer (4-6). It is well known that lncRNAs participate in cancer-associated pathways (7). Although an increasing number of lncRNAs have been reported to functionally regulate the metastatic behavior of GC cells, a large number of lncRNAs require identification, as $70-90 \%$ of the human genome transcribes RNA products, whereas protein-coding genes account for only $2 \%$ (8). Therefore, the present study focused on an lncRNA, AFAP1-antisense RNA 1 (AFAP1-AS1), which was first observed in esophageal cancer and acted as an oncogene, mediating effects in cell proliferation, migration and 
invasion (9). AFAP1-AS1 was also revealed to serve a crucial role in various types of cancer, including colorectal (10), breast (11), hepatocellular (12) and lung cancer (13). However, the expression and function of AFAP1-AS1 in the development of GC remains largely unknown.

The epithelial-mesenchymal transition (EMT) serves essential roles in carcinogenesis and tumor progression (14). The determinant step in EMT is local invasion through the epithelial basement membrane that modifies cell-cell and cell-matrix interactions (15). A previous study demonstrated that lncRNA also regulates the progress of EMT in GC (16); AFAP1-AS1 was revealed to participate in the progression of EMT and to facilitate metastasis in colorectal cancer (17). However, it is unclear whether AFAP1-AS1 is associated with the progression of EMT in GC.

\section{Materials and methods}

Patients and samples. All samples used in the present study were collected from the General Hospital of Chinese People's Liberation Army (Beijing, China) according to the protocols approved by the Ethics Review Board and written informed consent was obtained from all patients. A total of 80 pairs of tumor and matched normal tissues were included and these samples were registered between April 2011 and September 2013. The characterization of the samples is presented in Table I. The 36-month follow-ups of the $80 \mathrm{GC}$ patients (48 male, 32 female; median age, 58 years; age range, 32-76 years) was performed.

Cell culture. GC GES-1, AGS, BGC-823, MKN-45 and SGC-7901 cell lines, which were purchased from the American Type Culture Collection (Manassas, VA, USA), were used in the present study. AGS cells were cultured in F-12 medium (Hyclone; GE Healthcare, Chicago, IL, USA) supplemented with $10 \%$ fetal bovine serum (FBS; Gibco; Thermo Fisher Scientific, Inc., Waltham, MA, USA), and GES-1, BGC-823, MKN-45 and SGC-7901 cells were cultured in High-Glucose Dulbecco's modified Eagle's medium (DMEM), supplemented with $10 \% \mathrm{FBS}$ at $37^{\circ} \mathrm{C}$ in $5 \% \mathrm{CO}_{2}$.

Lentivirus infection. Two short hairpin RNAs (shRNAs), shRNA1 and shRNA2, which are vectors of AFAP1-AS1, were constructed and further packaged into lentiviruses by Shanghai GenePharma Co., Ltd., (Shanghai, China). A total of $10^{8}$ titer lentiviruses were obtained. Next, MKN-45 and SGC-7901 cells were infected with the lentiviruses at a multiplicity of infection at a ratio of 1 cell: 20 lentiviruses. The lentiviruses were added into the cell culture and softly shaken. A total of $48 \mathrm{~h}$ after infection, the decreased expression of AFAP1-AS1 regulated by the shRNAs were confirmed using reverse transcription-quantitative polymerase chain reaction (RT-qPCR), compared with a negative control.

RNA isolation. TRIzol reagent (Invitrogen; Thermo Fisher Scientific, Inc.) was used to extract the total RNA from frozen tissues and GC cells, according to the manufacturer's protocol. In brief, $1 \mathrm{mg}$ tissue or $1 \times 10^{6}$ cells were mixed with $1 \mathrm{ml}$ TRIzol reagent and $60 \mu \mathrm{l}$ nuclease-free $\mathrm{H}_{2} \mathrm{O}$ was added to re-suspend the RNA precipitation.
Table I. The clinical characterization of patients with GC.

\begin{tabular}{lc}
\hline Variable & Patients, $\mathrm{n}(\%)$ \\
\hline Sex & \\
Male & $48(60)$ \\
Female & $32(40)$ \\
Age, years & \\
$\geq 60$ & $42(52.5)$ \\
$\leq 60$ & $38(47.5)$ \\
Tumor location & \\
Body & $22(27.5)$ \\
Antrum & $31(38.8)$ \\
Cardia & $27(33.7)$ \\
Other & 0 \\
Histology & \\
Adenocarcinoma & $52(65)$ \\
Mucinous adenocarcinoma & $28(35)$ \\
Signet cell cancer & 0 \\
TNM stage & \\
I & $18(22.5)$ \\
II & $23(28.7)$ \\
III & $26(32.5)$ \\
IV & $13(16.3)$ \\
\end{tabular}

$\mathrm{n}=80$. GC, gastric cancer; TNM, Tumor-Node-Metastasis .

$R T-q P C R$. RT-qPCR was performed in two steps using two reaction kits, the PrimeScript RT reagent kit (Takara Biotechnology Co., Ltd., Dalian, China) and the Premix Ex Taq kit (Takara Biotechnology Co., Ltd.), according to the manufacturer's protocols. In the first step, cDNA was synthesized in $20 \mu \mathrm{l}$ volumes containing $10 \mu \mathrm{l}$ total RNA with genomic DNA removed, $4 \mu 15 \mathrm{X}$ reverse transcription buffer, $1 \mu 1$ Prime RT Enzyme, $1 \mu \mathrm{l}$ RT Primer mix and $4 \mu \mathrm{l}$ nuclease-free $\mathrm{H}_{2} \mathrm{O}$. In the second step, qPCR reactions were performed in $20 \mu \mathrm{l}$ volumes and run on a Bio-Rad IQ5 thermocycler (Bio-Rad Laboratories, Inc., Hercules, CA, USA). Thermocycling conditions were as follows: $94^{\circ} \mathrm{C}$ for $2 \mathrm{~min}$, followed by 40 cycles of $95^{\circ} \mathrm{C}$ for $15 \mathrm{sec}$ and $60^{\circ} \mathrm{C}$ for $30 \mathrm{sec}$. The primer sequences were as follows: AFAP1-AS1 forward, 5'-TCGCTCAATGGA GTGACGGCA-3' and reverse, 5'-CGGCTGAGACCGCTG AGAACTT-3'; GAPDH forward, 5'-GGTGGTCTCCTCTGA CTTCAACA-3' and reverse, 5'-TCTCTTCCTCTTGTGCTC TTGCT-3'. GAPDH mRNA was used as an internal reference. The expression of AFAP1-AS1 was analyzed using the $2^{-\Delta \Delta C q}$ method (18).

Proliferation assays. As MKN-45 and SGC-7901 cells had a high expression of AFAP1-AS1, these cells were used to analyze the loss-of-function of AFAP1-AS1. Then, these cells were seeded into 96-well pates at a density of 2,000 cells/well, respectively. The cell proliferation was analyzed using a Cell Counting kit-8 (CCK8; Beyotime Institute of Biotechnology, Haimen, China) at 0, 24, 48, 72 and 96 h, according to the manufacturer's protocols. The results were collected using an 

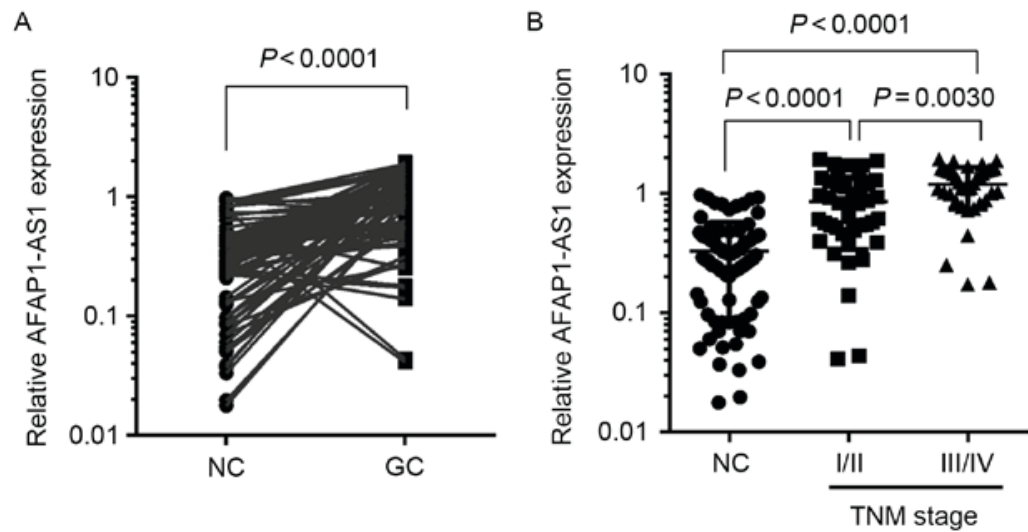

Figure 1. AFAP1-AS1 is overexpressed in GC tissues compared with matched NC tissues. (A) Expression of AFAP1-AS1 in 80 pairs of GC tissues and matched NC tissues. (B) Expression of AFAP1-AS1 in GC tissues at different TNM stages (stage I, n=18; stage II, n=23; stage III, n=26; and stage IV, $n=13$ ). GAPDH served as the internal reference. AFAP1-AS1, AFAP1-antisense RNA 1; GC, gastric cancer; NC, negative control; TNM, Tumor-Node-Metastasis.

ultraviolet spectrophotometer (Thermo Fisher Scientific, Inc.) at $450 \mathrm{~nm}$.

Migration and invasion assays. AFAP1-AS1-silenced MKN-45 and SGC-7901 cells at density of $5 \times 10^{4}$, and the same number of the parallel control cells, were used to perform migration or invasion assays. The migration and invasion of the cells were analyzed using a QCM Laminin Migration Assay kit (cat. no. ECM220; Merck KGaA, Darmstadt, Germany) and a Cell Invasion Assay kit (cat. no. ECM550; Merck KGaA), according to the manufacturer's protocols. FBS-free DMEM was used in the upper chamber and DMEM with 10\% FBS was used in the lower chamber. The number of cells transferred to the lower chamber were calculated using a light microscope (Olympus Corporation, Tokyo, Japan). The magnification was x10.

Western blot analysis. SGC-7901 cells with lentivirus containing negative control or shRNA-1 of lncRNA AFAP1-AS1, were seeded into 6-well plates prior to being collected for western blot analysis. The total proteins were extracted using RIPA buffer reagent (Thermo Fisher Scientific, Inc.), and the concentration of the lysate was determined using a BCA kit (Thermo Fisher Scientific, Inc.) according to the manufacturer's protocol. A total of $20 \mu \mathrm{g}$ of total proteins were separated using $12 \%$ SDS-PAGE gels and then transferred onto polyvinylidene fluoride (PVDF) membranes. Bovine serum albumin (3\%) solution (Beyotime Institute of Biotechnology) was used to block the PVDF membranes for $1 \mathrm{~h}$ at room temperature. Primary antibodies, E-cadherin (1:200; cat. no. 14472), N-cadherin (1:200; cat. no. 14215), GAPDH (1:1,000; cat. no. 5174$)$ and vimentin (1:500; cat. no. 5741) were purchased from Cell Signaling Technology, Inc. (Danvers, MA, USA) and were diluted using the Primary Antibody Dilution Buffer (Beyotime Institute of Biotechnology) according to the manufacturer's protocols. The PVDF membranes were incubated with the antibodies overnight at $4^{\circ} \mathrm{C}$. The secondary antibodies, HRP goat anti-mouse (cat. no. TA130003; 1:10,000) and HRP goat anti-rabbit (cat. no. TA140003; 1:10,000) (OriGene Technologies, Inc., Rockville, MD, USA) were diluted with the blocking solution (above mentioned) and then incubated with the PVDF membranes at room temperature for $1 \mathrm{~h}$. Finally, bound proteins were visualized using a SuperSignal
West Dura Extended Duration Substrate kit (Thermo Fisher Scientific, Inc.) and quantified using the ChemiDoc ${ }^{\mathrm{TM}}$ and ChemiDoc MP Imaging Systems with Image Lab ${ }^{\mathrm{TM}}$ Touch Software (Version 2.0) (Bio-Rad, California, USA).

Statistical analysis. All data were presented as mean \pm standard deviation. A two-tailed Student's t-test was used to compare the differences between two groups. One-way analysis of variance, followed by the Student-Newman-Keuls test, was used to analyze differences between more than two groups. Receiver operating characteristic (ROC) curve analysis was used for evaluating the effect of AFAP1-AS1 on differing GC from the adjacent normal tissues. The Youden index was used to analyze the optimal cut-off value. Survival analysis was used to evaluate the role of the expression of lncRNA AFAP1-AS1 in the survival time of patients with GC. $\mathrm{P}<0.05$ was considered to indicate a statistically significant difference. SPSS 17.0 (SPSS, Inc., Chicago, IL, USA) and GraphPad Prism 6.0 (GraphPad Software, Inc., La Jolla, CA, USA) software were used to perform all statistical analyses and to generate the graphs.

\section{Results}

AFAP1-AS1 expression is increased in the primary tumor tissue of $G C$ patients. The expression of AFAP1-AS1 was increased in various types of cancer $(10,12,17)$; however, its expression in GC was unclear. The expression of AFAP1-AS1 in 80 pairs of primary GC tissues and matched normal tissues was detected. The results revealed that the expression of AFAP1-AS1 was significantly higher in GC tissues than in the matched normal tissues $(\mathrm{P}<0.01$; Fig. 1A). Furthermore, the expression of AFAP1-AS1 was significantly increased in the tumor tissues of GC patients with Tumor-Node-Metastasis (TNM) stages (19) III and IV (P<0.01; Fig. 1B), indicating that the overexpression of AFAP1-AS1 may be associated with the aggressive progression of GC.

High expression of AFAP1-AS1 is associated with shorter survival times of patients with $G C$. To determine whether AFAP1-AS1 expression signature could serve as a biomarker for GC detection, ROC curve analysis was performed. The 
A

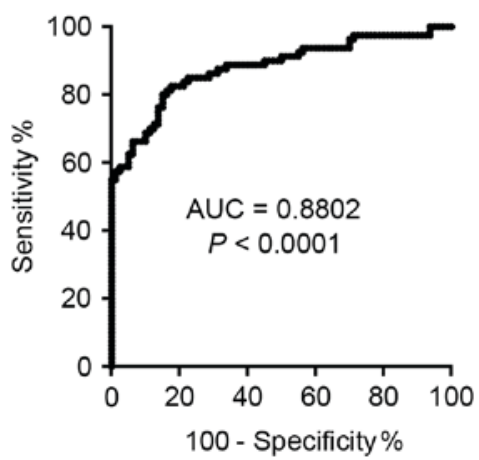

B

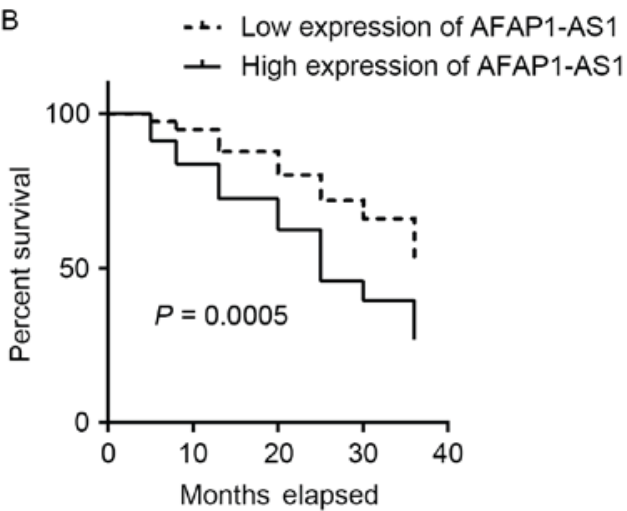

Figure 2. Expression of AFAP1-AS1 is associated with shorter survival times in patients with GC. (A) Receiver operating characteristic curve revealed that the expression signature of AFAP1-AS1 has an AUC of 0.8802 in distinguishing the GC tissues from the matched normal tissues (sensitivity, $81.25 \%$; specificity, 83.75\%). (B) Survival rate analysis revealed that the GC patients with high AFAP1-AS1 expression have lower survival rates. The high or low AFAP1-AS1 expression in GC tissues is determined using the cut-off value of 0.5040. AFAP1-AS1, AFAP1-antisense RNA 1; GC, gastric cancer; AUC, area under the curve.

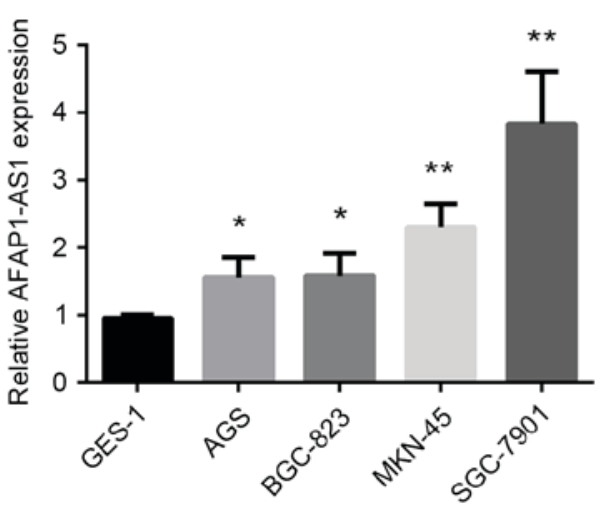

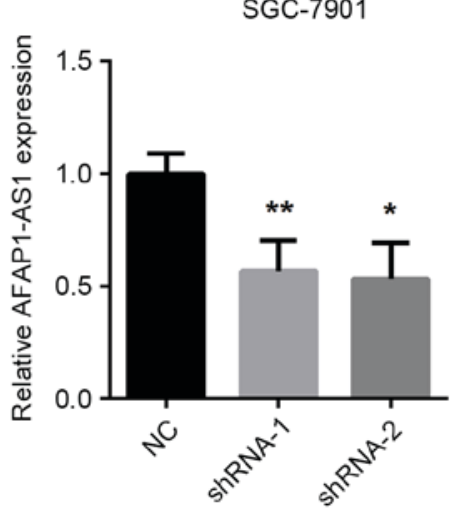

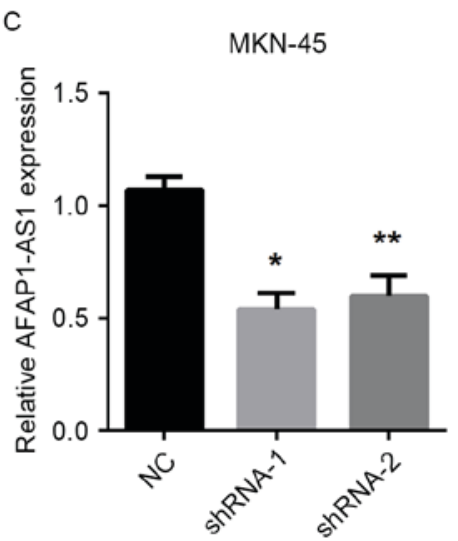

Figure 3. Specific short hairpin RNAs inhibit the expression of AFAP1-AS1. (A) The expression of AFAP1-AS1 in different GC cell lines. (B) The lentiviral vector containing shRNA-1 or shRNA-2 represses the expression of AFAP1-AS1 in SGC-7901 and MKN-45 cells. ${ }^{* *} \mathrm{P}<0.01$ and ${ }^{*} \mathrm{P}<0.05$. These experiments were repeated three times. AFAP1-AS1, AFAP1-antisense RNA 1; GC, gastric cancer; shRNA, short hairpin RNA.

results revealed that the expression signature of AFAP1-AS1 had potential as a diagnostic marker of GC when an optimal cut-off value of 0.5040 was selected (area under the curve, 0.8802; sensitivity, 81.25\%; specificity, 83.75\%; Fig. 2A). The association between the expression of AFAP1-AS1 and the survival time of patients with GC was also analyzed. As depicted in Fig. 2B, patients with GC that expressed high levels of AFAP1-AS1 exhibited a shorter survival time compared with those expressing low levels of AFAP1-AS1. These results indicated that the expression signature of AFAP1-AS1 might be a potential biomarker for the diagnosis and prognosis of GC.

Downregulation of AFAPI-AS1 is induced by specific shRNAs. To determine the biological role of AFAP1-AS1 in GC cells, the expression of AFAP1-AS1 was measured in five GC cells lines. As demonstrated in Fig. 3A, the expression of AFAP1-AS1 was significantly increased in SGC-7901 and MKN-45 cells compared with the other GC cells. Therefore, AFAP1-AS1 downregulation was induced in SGC-7901 and MKN-45 cells using lentiviruses containing specific shRNA-1 and shRNA-2 which would target AFAP1-AS1. The results revealed that shRNA-1 and shRNA-2 significantly decreased the expression of AFAP1-AS1 in the two cell lines, compared with negative control (NC) (Fig. 3B and C).

Downregulation of AFAP1-AS1 represses the proliferation, migration and invasion of GC cells in vitro. Using the aforementioned constructed cells, the effect of AFAP1-AS1 on GC cell proliferation was analyzed. The results revealed that shRNA-1 and shRNA-2 significantly decreased the proliferation of SGC-7901 and MKN-45 cells at on days 1, 2, 3 and 4 when compared with the negative control (NC) (Fig. 4A and B). Furthermore, the effect of AFAP1-AS1 on the migration and invasion of GC cells was also analyzed. As demonstrated in Fig. 5A and B, knockdown of AFAP1-AS1 expression significantly inhibited the migration of SGC-7901 and MKN-45 cells. These data indicated that AFAP1-AS1 might promote the proliferation and metastasis of cells in GC.

Downregulation of AFAP1-AS1 modulates the expression of EMT-associated genes. A previous study demonstrated that AFAP1-AS1 functions as an oncogene and promotes 
A

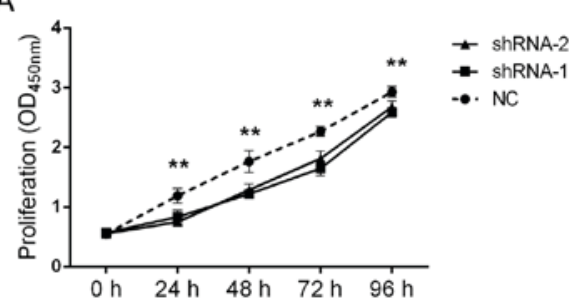

B

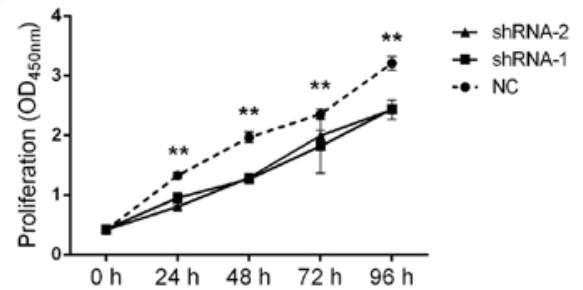

Figure 4. Downregulation of AFAP1-AS1 represses the proliferation of GC cells in vitro. (A) CCK8 assays revealed that AFAP1-AS1 silencing significantly inhibits the proliferation of (A) SGC-7901 (B) and MKN-45 cells compared with negative control (NC). ${ }^{* *} \mathrm{P}<0.01$ vs. NC. AFAP1-AS1, AFAP1-antisense RNA 1; GC, gastric cancer; shRNA, short hairpin RNA; NC, negative control; OD, optical density.

A

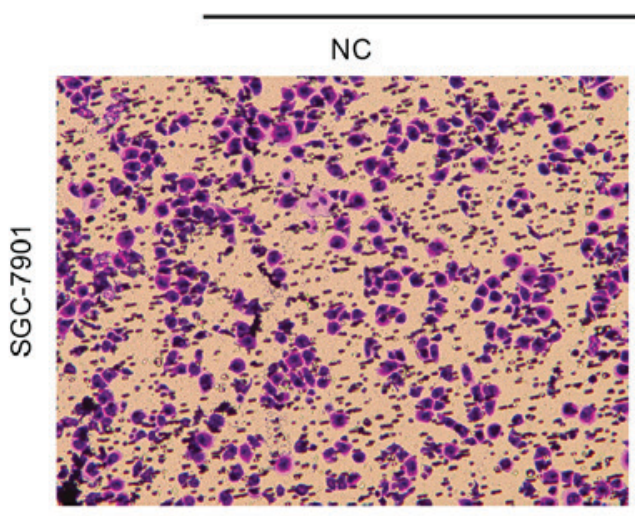

Migration
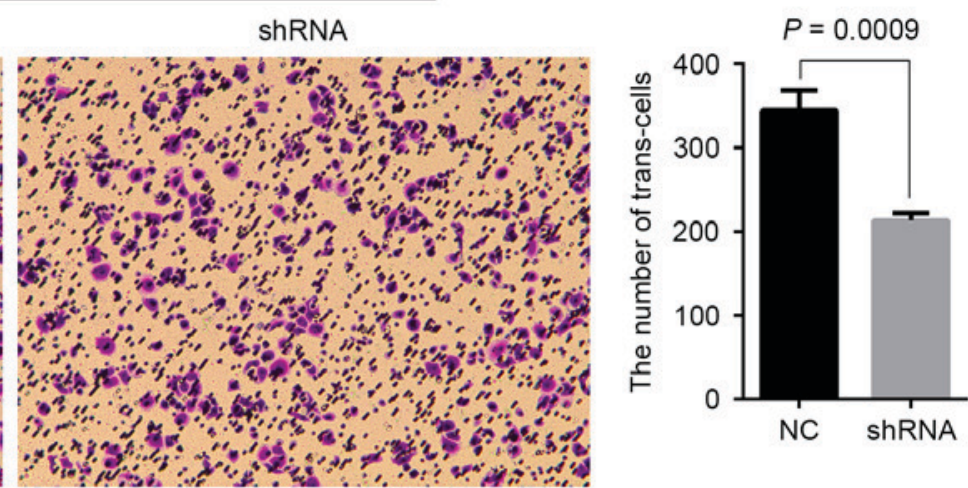

Invasion

B
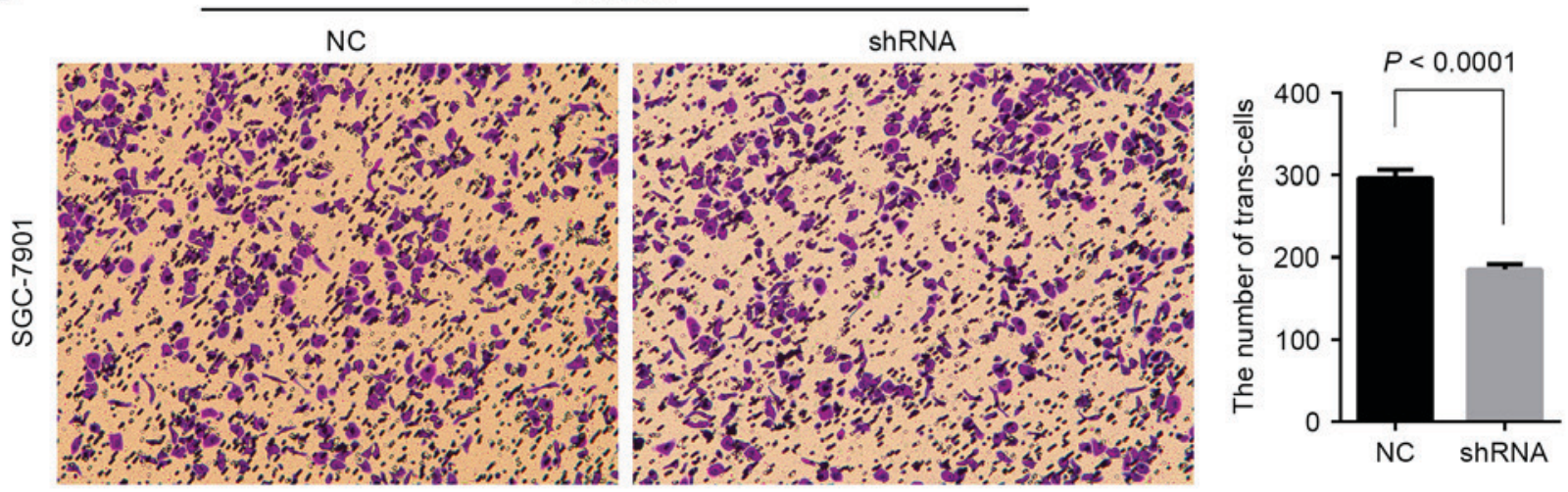

Figure 5. Downregulation of AFAP1-AS1 represses the migration and invasion of GC cells in vitro. SGC-7901 cells infected with lentivirus containing shRNA-1 have lower (A) migratory and (B) invasive abilities than those containing NC. These experiments were repeated three times. The magnification was x10. AFAP1-AS1, AFAP1-antisense RNA 1; GC, gastric cancer; shRNA, short hairpin RNA; NC, negative control.

GC cell metastasis (20). Therefore, the present study aimed to determine whether AFAP1-AS1 is involved in regulating EMT-associated genes, including E-cadherin, N-cadherin and vimentin. Western blot assays revealed that AFAP1-AS1 knockdown significantly increased the protein expression of $\mathrm{E}$-cadherin, whereas the protein expression of $\mathrm{N}$-cadherin and vimentin was significantly decreased (Fig. 6A and B). These results indicated that the AFAP1-AS1 induced the metastasis of GC cells through regulating EMT.

\section{Discussion}

The present study revealed that the expression of AFAP1-AS1 was increased in the GC tissues when compared to the matched normal tissues, and increased levels were associated with a shorter survival time in patients with GC. Furthermore, the expression signature of AFAP-AS1 was revealed to have the ability to distinguish GC tissues from matched normal tissues. The biological role of AFAP1-AS1 in GC cells was also determined and these data revealed that the downregulation of AFAP1-AS1 inhibited the proliferation and migration of GC cells.

AFAP1-AS1 was first identified in Barrett's esophagus and esophageal adenocarcinoma; its overexpression promoted cell proliferation and colony-forming ability, inhibit apoptosis, and enhance cellular migration and invasion in esophageal adenocarcinoma (9). The overexpression of AFAP1-AS1 has also been observed in various types of cancer, and is involved in the 

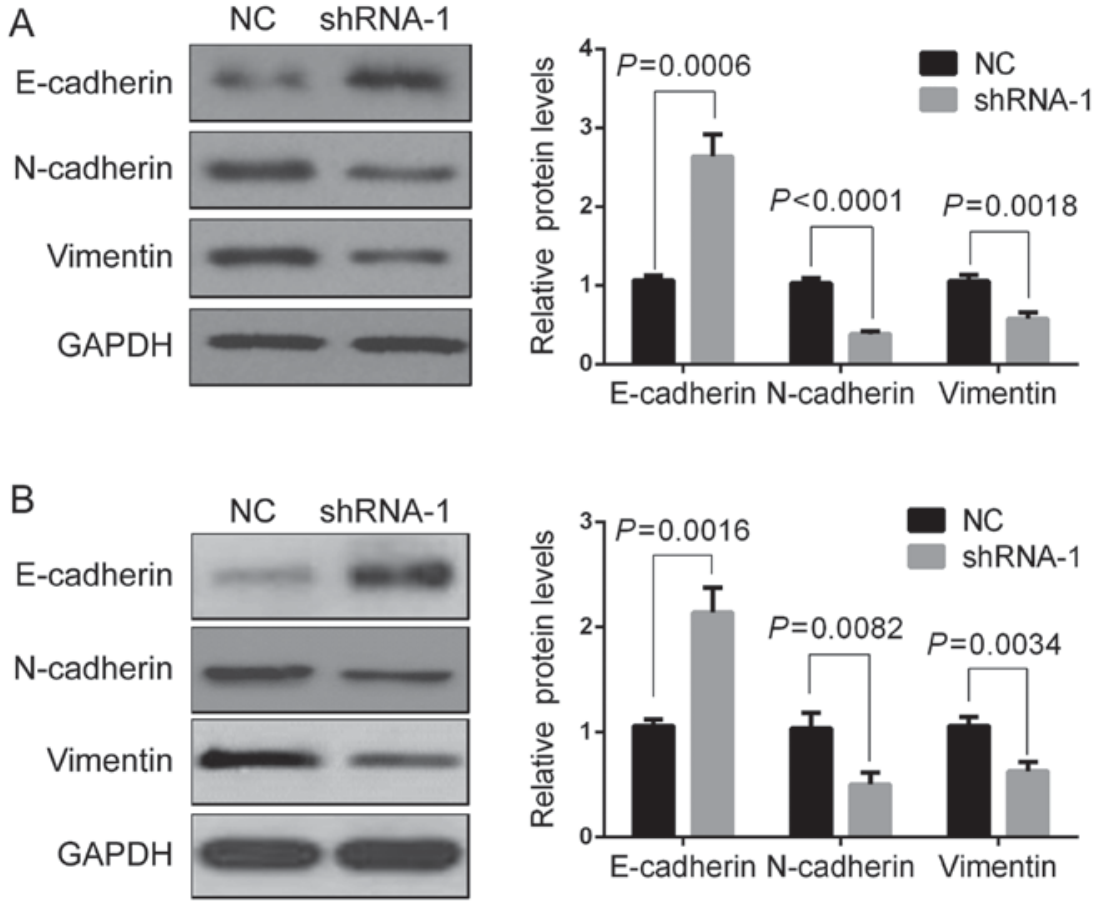

Figure 6. Downregulation of AFAP1-AS1 modulates the protein levels of epithelial-mesenchymal transition-associated genes. The protein level of E-cadherin is significantly increased, and the protein levels of N-cadherin and vimentin are significantly decreased in (A) SGC-7901 and (B) MKN-45 cells, which are infected with lentivirus containing shRNA-1 compared with those containing NC. GAPDH serves as internal reference. These experiments were repeated three times. AFAP1-AS1, AFAP1-antisense RNA 1; GC, gastric cancer; shRNA, short hairpin RNA; NC, negative control.

aggressive progression of cancer $(12,21-23)$. However, in GC, the expression and function of AFAP1-AS1 remains largely unclear. The present study detected the expression of AFAP1-AS1 in GC tissues and matched normal tissues, and observed that the expression of AFAP1-AS1 was also increased the GC tissues, a process that is associated with the aggressive progression of GC. Furthermore, the results of the present study demonstrated that the overexpression of AFAP1-AS1 was associated with shorter survival times in patients with GC. These observations indicated that the expression signature of AFAP1-AS1 may be a potential biomarker for the prognosis of patients with GC. Evidence has demonstrated that AFAP1-AS1 serves an oncogenic function in tumor development $(17,24,25)$. The results of the present study also indicated that a decrease in AFAP1-AS1 expression suppressed the proliferation, migration and invasion of GC cells, indicating that AFAP1-AS1 has a strong oncogenic function.

E-cadherin, a transmembrane glycoprotein of the type I cadherin superfamily, is the primary molecule in the adherent junctions in epithelial cells (14). The extracellular domain of E-cadherin forms dimers and interacts with the dimers of E-cadherin on the membrane of a neighboring cell; its intracellular domain is linked to a protein complex and is involved in intracellular actin filaments network (14). In contrast to epithelial cells, mesenchymal cells primarily express $\mathrm{N}$-cadherin, which has a dominant effect in cell-cell interactions. $\mathrm{N}$-cadherin enhances the motility of tumor cells via the destabilization of the cell-cell adhesion complex (26). The present study revealed that the inhibition of AFAP1-AS1 expression significantly increased the level of E-cadherin protein expression and decreased that of $\mathrm{N}$-cadherin in $\mathrm{GC}$ cells, indicating that AFAP1-AS1 participates in the regulation of the EMT progress in GC cells.
In the present study, the number of available clinical specimens was relatively small. Although the biological function of AFAP1-AS1 in GC cells was identified and its mechanism was investigated, the exact target of AFAP1-AS1 remains largely unclear. Additionally, the role of AFAP1-AS1 in the progress of $\mathrm{GC}$ requires further clarification in vivo. Additionally, more clinical samples are required to evaluate the differential ability of AFAP1-AS1 in the diagnosis of GC, and the exact mechanism of AFAP1-AS1 in GC cells requires identification.

In conclusion, the present study revealed that the overexpression of AFAP1-AS1 was associated with the shorter survival times of patients with GC, and that it promotes the proliferation, migration and invasion of GC cells, partially through regulating the EMT process, indicating that AFAP1-AS1 may serve as a novel biomarker and therapeutic target for the diagnosis and treatment of GC.

\section{Acknowledgements}

Not applicable.

\section{Funding}

The present study was supported by grants from the National Nature Science Foundation of China (grant nos. 81272698, 81672319 and 81602507).

\section{Availability of data and materials}

The datasets generated and analyzed in the present study are included in the published article. 


\section{Authors' contributions}

$\mathrm{HZ}, \mathrm{KZ}$, and $\mathrm{TW}$ performed the experiments. JC, HX, YW, and YS collected the patient samples. XZ analyzed the data. BW and LC designed the present study and wrote this paper.

\section{Ethics approval and consent to participate}

All samples used in the present study were collected from the General Hospital of Chinese People's Liberation Army (Beijing, China) according to the protocols approved by the Ethics Review Board and written informed consent was obtained from all patients.

\section{Consent for publication}

Study participants provided consent for the data to be published.

\section{Competing interests}

The authors declare that they have no competing interests.

\section{References}

1. Chen W, Zheng R, Baade PD, Zhang S, Zeng H, Bray F, Jemal A, Yu XQ and He J: Cancer statistics in China, 2015. CA Cancer J Clin 66: 115-132, 2016.

2. Ushijima T and Sasako M: Focus on gastric cancer. Cancer Cell 5: 121-125, 2004.

3. ENCODE Project Consortium: An integrated encyclopedia of DNA elements in the human genome. Nature 489: 57-74, 2012.

4. Glover AR, Zhao JT, Ip JC, Lee JC, Robinson BG, Gill AJ, Soon PS and Sidhu SB: Long noncoding RNA profiles of adrenocortical cancer can be used to predict recurrence. Endocr Relat Cancer 22: 99-109, 2015.

5. Yuan JH, Yang F, Wang F, Ma JZ, Guo YJ, Tao QF, Liu F, Pan W, Wang TT, Zhou CC, et al: A Long Noncoding RNA activated by TGF- $\beta$ promotes the invasion-metastasis cascade in hepatocellular carcinoma. Cancer Cell 25: 666-681, 2014.

6. Liu B, Sun L, Liu Q, Gong C, Yao Y, Lv X, Lin L, Yao H, Su F, Li D et al: A cytoplasmic NF- $\mathrm{BB}$ interacting long noncoding RNA Blocks I $\kappa \mathrm{B}$ phosphorylation and suppresses breast cancer metastasis. Cancer Cell 27: 370-381, 2015.

7. Schmitt AM and Chang HY: Long noncoding RNAs in cancer pathways. Cancer Cell 29: 452-463, 2016.

8. Zeng S, Xiao YF, Tang B, Hu CJ, Xie R, Yang SM and Li BS: Long noncoding RNA in digestive tract cancers: function, mechanism and potential biomarker. Oncologist 20: 898-906, 2015.

9. Wu W, Bhagat TD, Yang X, Song JH, Cheng Y, Agarwal R, Abraham JM, Ibrahim S, Bartenstein M, Hussain Z, et al: Hypomethylation of noncoding DNA regions and overexpression of the long noncoding RNA, AFAP1-AS1, in Barrett's esophagus and esophageal adenocarcinoma. Gastroenterology 144: 956-966.e4, 2013.

10. Wang F, Ni H, Sun F, Li M and Chen L: Overexpression of IncRNA AFAP1-AS1 correlates with poor prognosis and promotes tumorigenesis in colorectal cancer. Biomed Pharmacother 81: $152-159,2016$
11. Yang F, Lyu S, Dong S, Liu Y, Zhang X and Wang O: Expression profile analysis of long noncoding RNA in HER-2-enriched subtype breast cancer by next-generation sequencing and bioinformatics. Onco Targets Ther 9: 761-772, 2016.

12. Zhang JY, Weng MZ, Song FB, Xu YG, Liu Q, Wu JY, Qin J, Jin T and Xu JM: Long noncoding RNA AFAP1-AS1 indicates a poor prognosis of hepatocellular carcinoma and promotes cell proliferation and invasion via upregulation of the RhoA/Rac2 signaling. Int J Oncol 48: 1590-1598, 2016.

13. Zeng Z, Bo H, Gong Z, Lian Y, Li X, Li X, Zhang W, Deng H, Zhou M, Peng S, et al: AFAP1-AS1, a long noncoding RNA upregulated in lung cancer and promotes invasion and metastasis. Tumour Biol 37: 729-737, 2016.

14. Voulgari A and Pintzas A: Epithelial-mesenchymal transition in cancer metastasis: mechanisms, markers and strategies to overcome drug resistance in the clinic. Biochim Biophys Acta 1796: 75-90, 2009.

15. Mathias RA and Simpson RJ: Towards understanding epithelial-mesenchymal transition: a proteomics perspective. Biochim Biophys Acta 1794: 1325-1331, 2009.

16. Zhou H, Wang F, Chen H, Tan Q, Qiu S, Chen S, Jing W, Yu M, Liang C, Ye S and Tu J: Increased expression of long-noncoding RNA ZFAS1 is associated with epithelial mesenchymal transition of gastric cancer. Aging (Albany NY) 8: 2023-2038, 2016.

17. Han X, Wang L, Ning Y, Li S and Wang Z: Long non-coding RNA AFAP1-AS1 facilitates tumor growth and promotes metastasis in colorectal cancer. Biol Res 49: 36, 2016.

18. Livak KJ and Schmittgen TD: Analysis of relative gene expression data using real-time quantitative PCR and the 2(-Delta Delta C (T)) method. Methods 25: 402-408, 2001.

19. Sano T, Coit DG, Kim HH, Roviello F, Kassab P, Wittekind C, Yamamoto Y and Ohashi Y: Proposal of a new stage grouping of gastric cancer for TNM classification: International gastric cancer association staging project. Gastric Cancer 20: 217-225, 2017.

20. Bo H, Gong Z, Zhang W, Li X, Zeng Y, Liao Q, Chen P, Shi L, Lian Y, Jing Y, et al: Upregulated long non-coding RNA AFAP1-AS1 expression is associated with progression and poor prognosis of nasopharyngeal carcinoma. Oncotarget 6: 20404-20418, 2015.

21. Li Q, Dai Y, Wang F and Hou S: Differentially expressed long non-coding RNAs and the prognostic potential in colorectal cancer. Neoplasma 63: 977-983, 2016.

22. Bo H, Gong Z, Zhang W, Li X, Zeng Y, Liao Q, Chen P, Shi L, Lian Y, Jing Y, et al: Upregulated long non-coding RNA AFAP1-AS1 expression is associated with progression and poor prognosis of nasopharyngeal carcinoma. Oncotarget 6: 20404-20418, 2015.

23. Ye Y, Chen J, Zhou Y, Fu Z, Zhou Q, Wang Y, Gao W, Zheng S, Zhao X, Chen T and Chen R: High expression of AFAP1-AS1 is associated with poor survival and short-term recurrence in pancreatic ductal adenocarcinoma. J Transl Med 13: 137, 2015.

24. Luo HL, Huang MD, Guo JN, Fan RH, Xia XT, He JD and Chen XF: AFAP1-AS1 is upregulated and promotes esophageal squamous cell carcinoma cell proliferation and inhibits cell apoptosis. Cancer Med 5: 2879-2885, 2016.

25. Zhou XL, Wang WW, Zhu WG, Yu CH, Tao GZ, Wu QQ, Song YQ,Pan P and Tong YS: High expression of long non-coding RNA AFAP1-AS1 predicts chemoradioresistance and poor prognosis in patients with esophageal squamous cell carcinoma treated with definitive chemoradiotherapy. Mol Carcinog 55: 2095-2105, 2016

26. Nieman MT, Prudoff RS, Johnson KR and Wheelock MJ: $\mathrm{N}$-cadherin promotes motility in human breast cancer cells regardless of their E-cadherin expression. J Cell Biol 147: 631-644, 1999. 